\title{
AVALIAÇÃO DE FATORES PROGNÓSTICOS MORFOLÓGICOS DO CÂNCER COLORRETAL EM PACIENTES DO SERVIÇO DE ONCOLOGIA DO HOSPITAL ESCOLA ÁLVARO ALVIM
}

\author{
EVALUATION OF COLORECTAL CANCER MORPHOLOGICAL PROGNOSTIC FACTORS IN \\ PATIENTS FROM THE ONCOLOGY SERVICE OF HOSPITAL ESCOLA ÁLVARO ALVIM
}

\author{
Anna Luiza Soares Young ${ }^{1}$, Carolina Oliveira de Souza', Luíza de Miranda Póvoa', Maria Auxiliadora Peixoto \\ Peçanha². \\ ${ }^{1}$ Acadêmicas da Faculdade de Medicina de Campos; \\ ${ }^{2}$ Chefe da Cadeira de Patologia Geral da Faculdade de Medicina de Campos.
}

Hospital Escola Álvaro Alvim - R. Barão da Lagoa Dourada, 409 - Centro, Campos dos Goytacazes - RJ, 28035-211

Anna Luiza Soares Young

Endereço: Avenida Doutor Nilo Peçanha, número 1.

Telefone: (22) 998250255 - E-mail de correspondência: annaluizayoung@gmail.com

RESUMO

O câncer colorretal (CCR) é uma neoplasia de alta incidência em todo o mundo. Corresponde a terceira causa de morte oncológica em países industrializados. No Brasil, segundo as estatísticas do Instituto Nacional de Câncer (INCA), a neoplasia maligna colorretal é a quarta causa de óbitos por câncer em homens e a terceira causa em mulheres. Dessa forma sendo considerado um dos mais importantes problemas de saúde pública mundial. 0 presente estudo tem por objetivo comprovar os fatores que influenciam no prognóstico dessa doença, incluindo margem de ressecção circunferencial, invasão linfovascular e invasão perineural. Tais fatores são fundamentais para o melhor manejo desses pacientes, e consequentemente a redução da taxa de mortalidade.

Palavras-chaves: câncer colorretal, fatores prognósticos, margem de resseç̧ãocircunferencial, invasão linfovascular, invasão perineural.
ABSTRACT

Colorectal cancer (CRC) is a cancer of high incidence worldwide. It is identified as the third leading cause of cancer death in industrialized countries. In Brazil, according to statistics from the National Cancer Institute (INCA), a colorectal malignancy is the fourth leading cause of cancer deaths in men and the third leading cause in women. That is why is considered one of the most important public health problems in the world. The present study aims to prove the factors that influence the prognosis of this disease, including circumferential resection margin, lymphovascular invasion and perineural invasion. These factors are fundamental for the better management of patients, and, consequently, the reduction of the mortality rate.

Keywords: colorectal cancer, prognostic factors, circumferential resection margin, lymphovascular invasion, perineural invasion. 
INTRODUÇÃO

O câncer é um importante problema de saúde pública em países desenvolvidos e em desenvolvimento, sendo este o responsável por mais de seis milhões de óbitos a cada ano, representando cerca de $12 \%$ de todas as causas de morte no mundo ${ }^{1,2,3}$.

Sabe-se que o adenocarcinoma do cólon é a malignidade mais comum do trato gastrointestinal e é um dos principais contribuintes para a morbidade e mortalidade. A incidencia de câncer colorretal (CCR) atinge o pico entre 60 e 70 anos de idade. Múltiplos fatores podem se relacionar ao desenvolvimento de CCR, sendo estes: fatores ambientais, sexo masculino, predisposição genética e a obesidade. Outro fator associado ao desenvolvimento da patologia seria a alimentação, como por exemplo: a baixa ingestão de fibras vegetais inabsorvíveis e a alta ingestão de carboidratos refinados e gordura. Também são fatores de risco as doenças inflamatórias intestinais, como Retocolite Ulcerativa Crônica e Doença de Crohn, bem como doenças hereditárias, como Polipose Adenomatosa Familiar (PAF) e CCR Hereditário sem Polipose (HNPCC) ${ }^{4}$.

O objetivo do tratamento do CCR é conseguir a cura com o mínimo de complicações e de recidivas, preservando-se a qualidade de vida. Os melhores resultados dependem da precocidade do diagnóstico, do correto estadiamento, da boa técnica cirúrgica, bem como da assistência multidisciplinar que envolve a participação de profissionais especializados em oncologia, radioterapia, e cuidados nutricionais ${ }^{5}$.

O tratamento cirúrgico, a melhor modalidade de tratamento, pode ter indicação eletiva ou de urgência, e ter caráter curativo ou paliativo. Pode ser realizada nos casos de terapêutica do tumor primário, das recidivas locais, das metástases, além dos tumores metacrônicos. A ressecção cirúrgica do tumor representa ainda a única possibilidade de cura e deverá ser realizada da forma mais radical sempre que for considerada com possibilidade curativa, obedecendo aos princípios oncológicos já estabelecidos5. Preconiza-se a ressecção em monobloco, caso o tumor esteja aderido às estruturas ou aos órgãos vizinhos ${ }^{6}$.

Pela alta prevalência do $C C R$, torna-se imprescindível a análise de fatores prognósticos, como: margem de ressecção circunferencial (CRM), invasão linfovascular (LVI), e invasão perineural
(PNI). A CRM é a distância em milímetros entre o ponto mais profundo da invasão tumoral do câncer primário e a margem de ressecção no retroperitônio ou mesénterio. Quanto maior a distância, melhor o prognóstico. A LVI avalia a invasão de pequenos e grandes vasos pelo tumor primário, e, é um importante fator de mau prognóstico. A PNI, fornece evidência histológica de invasão de nervos ou espaços perineurais pelo tumor e possivelmente indica o pior prognóstico, similar ao da LVI ${ }^{7}$.

\section{OBJETIVOS}

O presente trabalho tem como objetivo realizar a confirmação dos fatores de prognóstico dos pacientes diagnosticados com câncer de cólon com a literatura médica, os quais são: margem de ressecção circunferencial, invasão linfovascular e invasão perineural. Para tal, realizou-se um estudo clínico em um município do Norte Fluminense do Estado do Rio de Janeiro. Assim, objetiva-se a colaborar o serviço de oncologia na traçagem de diretrizes ao realizar a identificação de um ou mais dos fatores de prognósticos analisados e apresentados.

\section{MÉTODO}

Este se trata de um estudo observacional, documental e transversal realizado no município de Campos dos Goytacazes - RJ, no período de fevereiro de 2019 a fevereiro de 2020, e este conta com aproximadamente 510.000 habitantes8. A amostra foi constituída por prontuários de todos os pacientes atendidos no setor de Oncologia do Hospital Escola Álvaro Alvim, neste mesmo município, contou com um recorte temporal de janeiro de 2016 a dezembro de 2018, que possuíam o diagnóstico de Adenocarcinoma colorretal.

Adotou-se o critério de amostragem não probabilístico por conveniência. O critério de inclusão utilizado foi o de avaliar todos pacientes internados pelo setor de Oncologia com diagnóstico de $C C R$, independente do sexo e comorbidades associadas. Houve recorte na amostra, uma vez que foram excluídos os pacientes menores de 18 anos, os que apresentaram alterações histopatológicas diferentes do adenocarcinoma, os que faleceram nos dois primeiros meses de pós-operatório por complicações cirúrgicas, e aqueles falecidos por causas não relacionadas à neoplasia.

\section{RESULTADOS}

Entre os anos de 2016 a 2018 foi obtida uma 
amostra de 345 pacientes diagnosticados com Neoplasia Colorretal internados no setor de oncologia do Hospital Escola Álvaro Alvim (Campos dos Goytacazes/RJ). Porém, 34 foram desconsiderados, uma vez que: 27 pacientes $(7,8 \%)$ apresentaram Adenoma; três pacientes (0,87\%) apresentaram Carcinoma Escamoso; e quatro pacientes $(1,15 \%)$ não apresentaram laudo histopatológico. Sendo assim, a amostra de pacientes que possuíam a Adenocarcinoma foi de 311 pessoas.

Dentre esses, apenas 75 pacientes preencheram os critérios de inclusão, representando aproximadamente $24 \%$ da amostra inicial. Os prontuários restantes, dos 236 pacientes, foram desconsiderados por apresentarem algum dos critérios de exclusão, como: faleceram nos dois primeiros meses de pós-operatório por complicações cirúrgicas; aqueles falecidos por causas não relacionadas à neoplasia; prontuários com informações incompletas; e pacientes transferidos para outro serviço hospitalar.

Dos 75 prontuários analisados, aproximadamente $27 \%$ eram pessoas com menos de 60 anos de idade. Com relação ao sexo dos pacientes em questão, $42,7 \%$ eram mulheres e $57,3 \%$ eram homens. Do total, $94,7 \%$ destes apresentaram o Adenocarcinoma moderadamente diferenciado; $4 \%$ eram do subtipo histológico bem diferenciado; e $1,3 \%$ era do subtipo indiferenciado/anaplásico.

Dos fatores prognósticos analisados, $8 \%$ apresentavam MRC comprometida; $20 \%$ apresentavam ILV; e 13,3\% apresentavam IPN.

Do total de pacientes analisados, $44 \%$ eram portadores metástase no momento do diagnóstico; e em $30,3 \%$ foram detectadas metástases à distância.

Dos pacientes analisados, $34,7 \%$ foram tratados cirurgicamente, por meio da ressecção da neoplasia.

Além da conduta cirúrgica, $42,7 \%$ dos pacientes receberam também a quimioterapia. $E$, em $22,7 \%$ foram aplicados o tratamento tríplice (cirurgia, quimioterapia e radioterapia).

Dentre os 75 pacientes abordados no estudo, dez evoluíram ao óbito, sendo seis mulheres $(60 \%)$ e quatro (40\%) homens. Observou-se que dos óbitos, duas pessoas (20\%) possíam menos de 60 anos.

Dentre os fatores prognósticos, $10 \%$ dos óbitos apresentaram margem circunferencial comprometida; $30 \%$ apresentaram invasão linfovascular; e $20 \%$ apresentaram invasão perineural. Todos que evoluíram com o óbito eram Adenocarcinoma moderadamente diferenciado.

Foi observado que $60 \%$ dos pacientes apresentavam metástase, sendo que dois terços desses portavam apenas metástase linfonodal e um terço apresentavam acometimento à distância.

Dentre os pacientes que foram ao óbito, $50 \%$ foram tratados com cirurgia e quimioterapia; $20 \%$ com cirurgia, quimioterapia e radioterapia; e $30 \%$ apenas cirurgia.

\section{DISCUSSÃO}

A presença ou ausência $L V I^{9},{ }^{10},{ }^{11}, 12, M R C$ comprometida ${ }^{13}, 14,15,16$, ou PNI ${ }^{18}, 19,20,21,22,23,24,{ }^{25}$ foram reconhecidas como fortes fatores prognósticos do CCR. Presumivelmente, a deteç̧ão de células cancerosas em vasos peritumorais ou intratumorais ou tecidos neurais, fornece um sinal de que o processo metastático começou e, portanto, é geralmente considerado um indicador de mau prognóstico. No entanto, ainda há poucos estudos que comprovam a real significância desses fatores no prognóstico dos pacientes com $\mathrm{CCR}^{26}$.

Ao analisar a prevalência dos fatores prognósticos estudados - MRC, LVI, PNI - observouse que $36 \%$ dos pacientes apresentaram um dos fatores comprometidos e desses, $22 \%$ evoluíram ao óbito. A PNI é um fator independente de recidiva tumoral e óbito ${ }^{27}$. Dentre os 10 pacientes que evoluíram a óbito, oito eram maiores de 60 anos, fato este que corrobora com outras pesquisas, pois este perfil de paciente tende a protagonizar a maioria dos casos de morte por $\mathrm{CCR}^{28}$.

Vale ressaltar que $60 \%$ dos óbitos apresentavam um dos fatores prognósticos incluídos (sem concomitância de fatores), destacando o impacto do comprometimento dessas variáveis para o desfecho dos casos. Também foi possível observar que dentre os pacientes que evoluíram ao óbito com algum fator prognóstico comprometido, $50 \%$ apresentava Invasão Linfovascular (ILV) na análise histopatológica; 33\% apresentava invasão perineural (IPN) e $16,7 \%$ apresentava margem de ressecção (MRC) comprometida.

A Sociedade Brasileira de Coloproctologia, em 2018, demonstrou que a invasão perineural está associada a um pior prognóstico do câncer 
colorretal, conforme demonstrado pela sobrevida global (Razão de risco (HR) = 1,30; IC 95\%: 1,13-1,50; $p<0,01$ ) e sobrevida livre de doença ( $H R=2,14$; IC 95\%: 2,06-2,22 $p<0,01) 29$. Outros estudos, como o realizado em Buffalo/NY, mostraram que o PNI foi independentemente associado à redução da sobrevida (HR 3,55, IC 95\% 1,78-7,09), e que LVI e PNI estão associados ao CCR avançado, uma vez que o PNI é um marcador independente de mau prognóstico para a sobrevivência em CCR30. Em adicional, um estudo realizado em Copenhagen em 2018, provou que linfócitos infiltrantes de tumor + CD3 e CD8 baixos na área invasiva estariam ambos relacionados ao prognóstico inferior de CCR em estágio II, e recomendam que qualquer um desses parâmetros seja considerado como fator de alto risco ${ }^{31}$.

Por fim, um ensaio realizado no Reino Unido, reuniu 259 pacientes. Destes, 138 (53,3\%) pacientes desenvolveram doença recorrente, dos quais 95 morreram. A duração média do acompanhamento nos pacientes restantes foi de 28 meses (variação: 12-96 meses). Houve associações significativas entre a recorrência e maior número de tumor $(P=0,002)$, presença de invasão perineural ( $P$ $=0,009)$ e ressecção de margem positiva (R1) $(P=$ 0,002 ). Em análise multivariada foi possível associar que todos os três fatores prognósticos são preditores independentes de sobrevida livre de doença. Assim, a presença de invasão perineural, múltiplos tumores e uma margem de ressecção comprometida foram associados à doença recorrente ${ }^{32}$.

Notou-se mais da metade dos apresentaram CCR eram homens, este resultado era esperado uma vez que o risco de desenvolvimento de câncer colorretal ao longo da vida é um pouco maior nos homens, $4,4 \%$, e para as mulheres $4,1 \%^{33}$.

Em suma, através do estudo foi possível correlacionar a presença de um dos fatores prognósticos estudados com a incidência de óbito na população acometida com CCR, e que dentre eles a LVI foi o fator predominante nos pacientes com pior desfecho, porém, de acordo com a literatura o fator mais agravante é o PNI.

Apesar de não ter sido o principal objetivo do trabalho, foi possível obter análise epidemiológica acerca do perfil de pacientes acometidos com CCR na amostra em questão. A título de exemplo: pacientes do sexo masculino foram mais acometidos pelo CCR, apesar dos pacientes do sexo feminino obterem pior desfecho, no estado de São Paulo, por exemplo, também apresenta este mesmo resultado34; pacientes acima de 60 anos apresentaram pior prognóstico, como era esperado34; a maioria dos óbitos está relacionado com algum grau de metástase, seja essa linfonodal ou a distância, resultado este encontrado também na literatura ${ }^{13},{ }^{35}$.

Devido às limitações inerentes a um estudo retrospectivo e ao tamanho relativamente pequeno da amostra dessa análise, o resultado encontrado requer maior investigação para se chegar a uma conclusão largamente aplicável. A ampliação de estudos e pesquisas que avaliem tais fatores prognósticos, garantindo maiores amostras e acompanhamento em longo prazo, seria capaz de assegurar maior compreensão dos pacientes diagnosticados com CCR, influenciando de forma positiva em seu prognóstico e tratamento.

\section{CONCLUSÃO}

Este se objetivou a realizar uma confirmação dos fatores de prognóstico dos pacientes diagnosticados com câncer de cólon em um estudo clínico comparado a literatura médica, tendo como fatores principais a margem de ressecção circunferencial, a invasão linfovascular e a invasão perineural.

Sabe-se que o CCR enquadra-se como uma neoplasia de alta incidência e prevalência no mundo, sendo esta a quarta, e no Brasil, a quinta, demonstrando assim a atualidade da pauta e a importância de elaborar estudos a respeito do tema em questão.

Apesar das limitações, através do estudo foi possível entender e reforçar os fatores que influenciam no prognóstico, além de estimar qual desses apresentam maior destaque na expectativa de vida dos pacientes acometidos, sendo comprovado na amostra analisada que o LVI foi o fator predominante nos pacientes com pior desfecho.

Com base ao que foi apresentado, pode-se concluir que o presente estudo atingiu aos objetivos propostos e tem a capacidade de corroborar para futuras pesquisas com maiores bancos de dados e, por conseguinte, contribuir para um tratamento individualizado e com maiores chances desfechos favoráveis. 


\section{REFERÊNCIAS}

1- Todaro M. Colon Cancer Stem Cells Dictate Tumor Growth and Resist Cell Death by Production of Interleukin-4.Cell Stem Cell. , v.1(4), p.389-402, 2007.

2-Kumar V, Abbas AK, Aster JC. Robbinspatologia básica. Elsevier Brasil, 2013.

3- Neves FJ, Mattos IE, Koifman RJ. Mortalidade por câncer de cólon e reto nas capitais brasileiras no período 1980-1997. Biblioteca Virtual de Saúde, Arq. Gastroenterol., v. 42(1), p. 63-70, 2005.

4- Abanto L, Horna R, Olivares G. Colorectal cancer in young adults: clinical and epidemiological features in the Peruvian population. Rev Gastroenterol, Peru, Apr-Jun; 37(2): 137-141. 2017.

5- FMUSP. Clínica Cirúrgica. São Paulo: Editora Manole, 2008.

6- Guerra MR, Gallo CVM, Mendonça GAS. Risco de câncer no Brasil: tendências e estudos epidemiológicos mais recentes. Revista Brasileira de Cancerologia (RBC), Rio de Janeiro,v.51, n.3, p. 227-234, 2005.

7- AJCC. Cancer Staging Manual 8th ed. Chicago: Editora Springer, 2017.

8- IBGE, Instituto Brasileiro de Geografia e Estatistica. Panorama de Campos dos Goytacazes, 2020. Disponível em: <https://cidades.ibge.gov.br/brasil/rj/campos-dos-goytacazes/panorama> Acessado em: 03/09/2020.

9- Ohlsson B, Breland U, Ekberg H, Graffner H, Tranberg KG. Follow-up after curative surgery for colorectal carcinoma. Randomized comparison with no follow-up. Dis Colon Rectum. 1995; 38(6):619-26.

10- Obrand DI, Gordon PH. Incidence and patterns of recurrence following curative resection for colorectal carcinoma. Dis Colon Rectum. 1997; 40(1):15-24.

11-Zlobec I, Lugli A. Prognostic and predictive factor in colorectal cancer. J Clin Pathol. 2008 May;61(5):561-569.

12- Compton CF, Burgart LJ, et al. Prognostic factors in colorectal cancer. College of American Pathologists Consensus Statement. Arch Pathol Lab Med. 1999;124:979-994.

13- Liang P, Hong JW, Ubukata H, Liu HR, Watanabe $Y$, Katano $M$, et al. Increased density and diameter of lymphatic microvessels correlate with lymph node metastasis in early stage invasive colorectal carcinoma. Virchows Arch. 2006 May; 448(5):570-575.

14- Compton C, Fenoglio-Preiser CM, Pettigrew N, Fielding LP. American Joint Committee on Cancer Prognostic Factprs Consensus Conference: Colore4ctal Working Group. Cancer. 2000 Apr 1;88(7): 1739-57.

15- Compton C. Colorectal Carcinoma: Diagnostic, Prognostic, and Molecular Features. Mod Pathol. 2003; 16(4): 376388.

16- COMPTON C. Prognostic factors in cancer. 3rd ed: Jhon Wiley \& Sons; 2006.

17-Fisher B, Redmond C, Fisher ER, Bauer M, Wolmark N, Wickerham DL, et al. Ten-years results of a randomized clinical trial comparing radical mastectomy and total mastectomy with or without radiation. N Engl J Med 1985; 312:974-681.

18- Jass JR, Atkin WS, Cuzick J, Bussey HJ, Morson BC, Northover JM, et al. The grading of reactal cancer: historical perspectives and a multivariate analysis of 477 cases. Histopathology. 1986 May; 10(5): 437-459.

19- Compton C, Fenoglio-Preiser CM, Pettigrew N, Fielding LP. American Joint Committee on Cancer Prognostic Factprs Consensus Conference: Colore4ctal Working Group. Cancer. 2000 Apr 1;88(7): 1739-57.

20- Halvorsen S E. Association between invasiveness, inflammatory reaction, desmoplasia and survi8val in colorectal cancer. J Clin Pathol. 1989 Feb; 42(2): 162-166.

21- Santo GFE, Aguilar-Nascimento JE, Kishimalll MO. Correlação de fatores anatomopatológicos com a sobrevida de pacientes operados por adenocarcinoma colorretal. Rev. Col. Bras. Cir. vol.35 no.3 Rio de Janeiro May/June 2008.

22- Priolli DG; Cardinalli IA; Piovesan H; Margarido NF; Martinez CAR. Proposta para estadiamento do câncer colorretal baseada em critérios morfofuncionais. Correlação com níveis séricos do antígeno carcinoembrionário. Rev bras. coloproctol. vol. 27 no.4 Rio de Janeiro Oct./Dec. 2007.

23- Dawson $\mathrm{H}$, et al. Tyrosine kinase receptor B (TrkB) expression in colorectal cancers highlights anoikis resistance as a survival mechanism of tumour budding cells. Histopathology, v. 66, n. 5, p. 715-725, 2015.

24- Lugli A, et al. Recommendations for reporting tumor budding in colorectal cancer based on the International Tumor Budding Consensus Conference (ITBCC) 2016. Modern Pathology, n. February, p. 1-13, 2017.

25-Sagaert, X. Prognostic biomarkers in colorectal cancer: where do we stand? Virchows Archiv: an international journal of pathology, v. 464, n. 3, p. 379-91, 2014.

26- Huh JW, et al. Prognostic significance of lymphovascular or perineural invasion in patients with locally advanced colorectal cancer. American Journal of Surgery, Elsevier Inc, v. 206, ed. 5, p. 758-763, novembro 2013

27- Mulcahy HE, Toner M, Patchett SE, Daly L, O'Donoghue DP. Identifying stage B colorectal cancer patients at high risk of tumor recurrence and death. Dis Colon Rectum. 1997; 40(3):326-31. 
28- Silva AA, Cordeiro HM, Novaes MCC, Sousa MBS, Magalhães RPM, Oliveira MVM. Morbimortalidade hospitalar por câncer colorretal no Brasil, no período de 2008 a 2016. Revista Eletrônica Acervo Científico / Electronic Journal Scientific Collection, 2019, Vol. 5 | e939 | DOI: https://doi.org/10.25248/reac.e939.2019.

29- Murugesan JR, Murugesan SP, Yip J, Hitos K, Fulham S, Engel A. Meta-analysis - perineural invasion as prognostic factor in rectal cancer. j coloproctol (rio j). 2019;39(1):74-80.

30- Al-Sukhni E, Attwood K, Gabriel EM, LeVea CM, Kanehira K, Nurkin SJ. Lymphovascular and perineural invasion are associated with poor prognostic features and outcomes in colorectal cancer: A retrospective cohort study. International Journal of Surgery 37 (2017) 42-49.

31- Eriksen AC, Sørensen FB, Lindebjerg J, Hager H, Christensen RP, Kjær-Frifeldt S, Hansen TF. The Prognostic Value of TumorInfiltrating lymphocytes in Stage II Colon Cancer. A Nationwide Population-Based Study. Translational Oncology (2018) 11, 979-987.

32- Gomez D, Zaitoun AM, Rosa A, Hossaini S, Beckingham IJ, Brooks A, Cameron IC. Critical review of the prognostic significance of pathological variables in patients undergoing resection for colorectal liver metastases. HPB 2014, 16, 836-844.

33- Instituto Oncoguia. Tipos de Câncer - Estatítica para cêncer colorretal, 2020. Disponível em: <http://www.oncoguia.org.br/conteudo/estatistica-para-cancer-colorretal/7284/178/> Acessado em 04/09/2020. 34- INCA, Instituto do Câncer Rio Preto, Câncer Colorretal - Introdução ao Câncer Colorretal, 2020. Disponível em: <https://incariopreto.com.br/cancer-colorretal-2/>Acessado em:03/09/2020.

35- Backus HH, Van Groeningen CJ, Vos W, Dukers DF, Bloemena E, Wouters D, et al. Differential expression of cell tumors, and liver metastases. J Clin Pathol. 2002 Mar;55(3):206-211. 